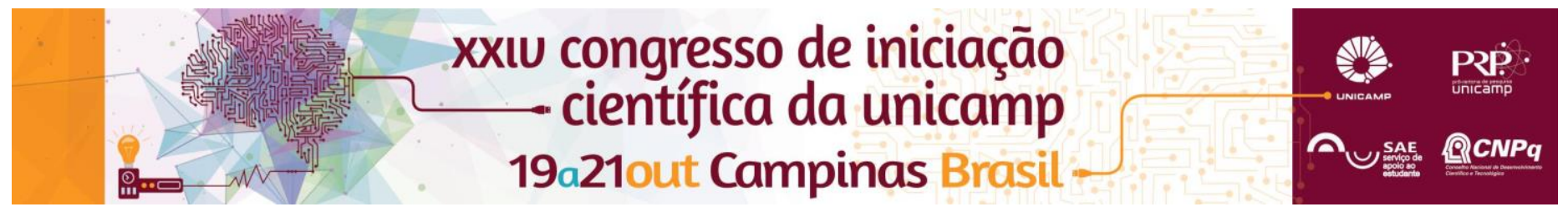

\title{
Adiposity of isolated adipocytes from fed hypertensive rats with the same age.
}

\section{Sandrine G. Gouvêa*, Gustavo T. Costa; Larissa Y. Ishizu, Ana G. Conceição-Vertamatti, Filipy Borghi, Dora M. Grassi Kassisse.}

\section{Abstract}

There are studies showing the relationship between high adiposity and hypertension, none about the lipodystrophy in spontaneously hypertensive rats. We analyzed body weight, food intake, white fat pads and morphometry of isolated adipocytes from fed rats in two different hypertensive rat models, induced and genetic. Hypertensive models were lighter, intake less food and shower lower adiposity than controls, and the genetic control was heavier and consumed less food than Wistar.

Key words: Hypertension, adiposity, rats.

\section{Introduction}

There are studies in the literature showing the relationship between hypertension and adiposity ${ }^{1}$ however, there are no studies evaluating the lipodystrophy in spontaneously hypertensive rats. The aim of this work is evaluate body weight, food intake, white fat pads and morphometry of isolated adipocytes from fed rats in two different hypertensive rat models, induced $(\mathrm{HI})$ and genetic $(\mathrm{HG})$. Their respective controls are Wistar (W) and Wistar Kyoto (WKY). HI rats were treated with L-Name per os ${ }^{2}$. Statistical analysis: As some results presented no normal distribution, we performed non-parametric test, as MannWhitney or Anova followed by Dunn's. Anova followed by Tukey or Student $t$-test applied to normal tests. Costa et al., $2015^{2}$ performed similar assays in fasted rats.

\section{Results and Discussion}

Hypertensive models were lighter, intake less food and shower lower adiposity than controls, and the genetic control was heavier and consumed less food than Wistar.

Figure 1- Body weight $(\mathrm{g})$ and food intake $(\mathrm{g} / \mathrm{g})$ per day of W, HI, WKY and HG rats in different ages

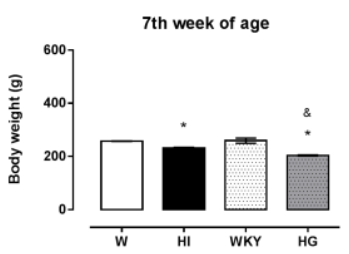

10th week of age
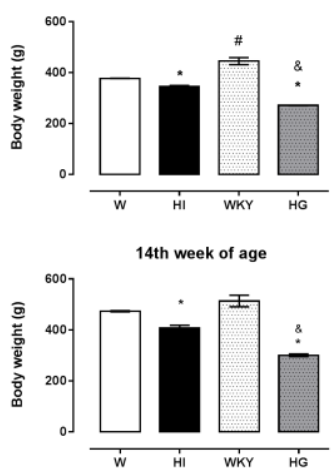
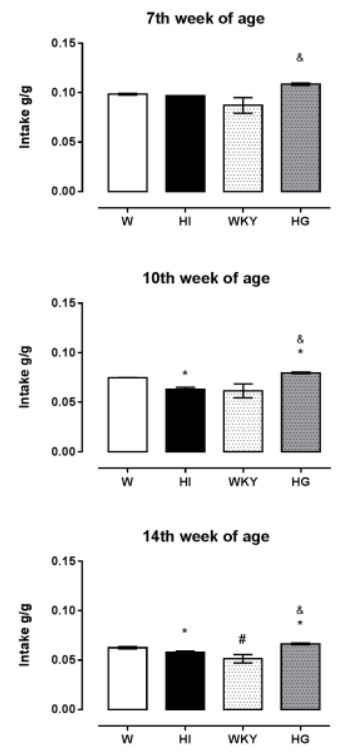

Figure 2- Area and diameter of isolated adipocytes from epididymal (a), perirenal (b) and mesenteric (c) depots. 1Wistar; 2- HI; 3-WKY and 4 HG.

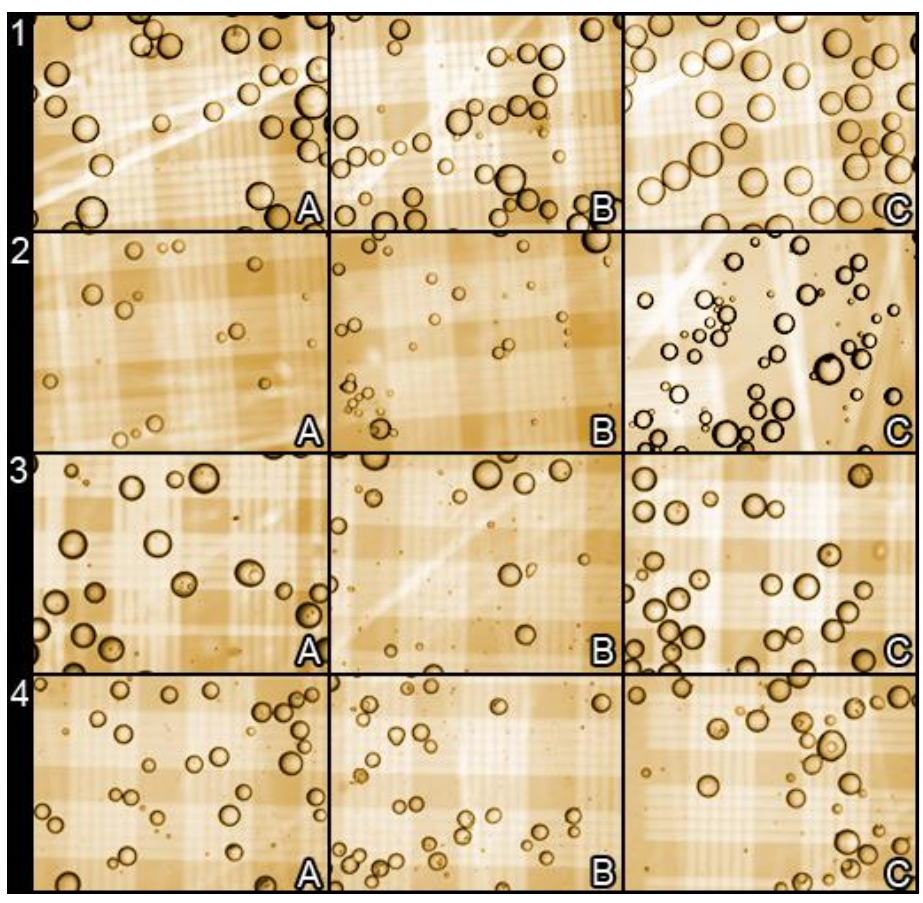

\section{Conclusions}

The fed status interfered in morphometric results, and the peri-renal and mesenteric differences showed by Costa et al., 2015 were not observed in this work.

\section{Acknowledgement}

FAPESP, FAEPEX, CAPES and PIBIC-CNPq

${ }^{1}$ Kishi, T and Kirooka, Y. Int. J. of Hypertension. 2013, p. 1-7.

${ }^{2}$ Costa et al., 2015. Morphometry of isolated adipocytes from rats of two models of hypertension. XXIII Congresso de Iniciação Cientírifa da Unicap, Vol1, ano 2015: 10.19146/pibic-2015-37344

${ }^{*} p<0.05$ hypertensive vs control $\# p<0.05$ WKY vs W $\& \mathrm{p}<0.05 \mathrm{HG}$ vs $\mathrm{HI}$ 